\title{
Síndrome metabólico y otros factores asociados a gonartrosis
}

\author{
Sergio Charles-Lozoya', Joaquín Darío Treviño-Báez², Jesús Alejandro Ramos-Rivera', \\ Jesús María Rangel-Flores ${ }^{3}$, Juan Carlos Tamez-Montes ${ }^{4}$ y Jesús Miguel Brizuela-Ventura ${ }^{1}$ \\ ${ }^{1}$ Servicio de Artroplastia de Rodilla, Unidad Médica de Alta Especialidad (UMAE), Hospital de Traumatología y Ortopedia No. 21, Instituto Mexicano \\ del Seguro Social (IMSS), 2Jefatura de División de Investigación en Salud de la UMAE, Hospital de Ginecología y Obstetricia No. 23, IMSS, \\ ${ }^{3}$ Dirección de Educación e Investigación en Salud; ${ }^{4}$ Dirección General. UMAE Hospital de Traumatología y Ortopedia No. 21, IMSS. Monterrey, \\ N.L. México
}

\section{Resumen}

Objetivo: Estimar si existe asociación del síndrome metabólico (SM) y otros potenciales factores de riesgo con la gonartrosis. Método: Estudio transversal comparativo de 310 pacientes evaluados por patología de rodilla agrupados en pacientes con gonartrosis y sin ella. Se recolectaron variables sociodemográficas, antropométricas y de laboratorio. La gonartrosis fue definida con un grado $\geq 2$ en la escala radiológica de Kellgren-Lawrence y el SM según la International Diabetes Federation. EI análisis bivariado fue con odds ratio, y el multivariado mediante regresión logística. Resultados: La prevalencia de SM en pacientes con gonartrosis fue del $79.9 \%$, estadísticamente mayor que en los pacientes sin gonartrosis $(p=0.001)$. Otros factores estadísticamente más frecuentes en los pacientes con gonartrosis fueron diabetes mellitus $(p=0.02)$ e hipertensión arterial $(p=0.02)$. En el análisis multivariado, el SM se asoció con mayor gonartrosis $(p=0.003), y$ con menor presencia de ella las lipoproteínas de alta densidad $(p=0.02$ ). Conclusiones: El SM y otras alteraciones relacionadas con él se encuentran asociados a la gonartrosis, y su control pudiera prevenir la aparición de esta enfermedad.

PALABRAS CLAVE: Prevalencia. Osteoartritis. Rodilla. Síndrome metabólico.

\begin{abstract}
Objective: To evaluate whether an association exists between gonarthrosis and metabolic syndrome $X(M S)$ as well as other potential risk factors. Method: Comparative cross-sectional study of 310 patients evaluated by pathology of knee grouped in patients with gonarthrosis and without it. Sociodemographic, anthropometric and laboratory data was obtained. Gonarthrosis was defined as a $\geq 2$ score in Kellgren-Lawrence radiological scale, and MS was assessed using the International Diabetes Federation criteria. Odds ratio and logistic regression were used for bivariate and multivariate analysis respectively. Results: The prevalence of MS in patients who had gonarthrosis was $79.9 \%$, statistically higher than in patients without gonarthrosis $(p=0.001)$. Other factors that had a statistically higher frequency in this group included diabetes mellitus $(p=0.02)$ and hypertension $(p=0.02)$. Multivariate analysis revealed MS had an association with a higher prevalence of gonarthrosis $(p=0.003)$, while high density lipoproteins $(p=0.02)$ was associated with a lower prevalence. Conclusions: $M S$ and its related alterations are associated to gonarthrosis; their adequate control could prevent patients from developing the disease.
\end{abstract}

KEY WORDS: Prevalence. Osteoarthritis. Knee. Metabolic syndrome.

\author{
Correspondencia: \\ Joaquín Darío Treviño-Báez \\ Avda. Constitución y Avda. Félix U. Gómez, \\ $\mathrm{s} / \mathrm{n}, 10^{\circ}$ piso \\ Col. Centro \\ C.P. 64010 , Monterrey, N.L., México \\ E-mail: joaquindtb @yahoo.com.mx
}

Fecha de recepción en versión modificada: 08-01-2017

Fecha de aceptación: 15-05-2017

DOI://dx.doi.org/10.24875/GMM.17002892
Gac Med Mex. 2017;153:775-780

Contents available at PubMed www.gacetamedicademexico.com 


\section{Introducción}

La osteoartritis de rodilla o gonartrosis es uno de los padecimientos más comunes de las articulaciones, y se ha asociado con factores mecánicos, envejecimiento, sexo femenino, obesidad y últimamente con alteraciones metabólicas ${ }^{1}$. La gonartrosis sintomática está presente en el $10 \%$ de los hombres y en el $13 \%$ de las mujeres de 60 años o más ${ }^{2}$. Se ha reportado una prevalencia de osteoartritis asociada a síndrome metabólico (SM) del $59 \%$ en una muestra de representatividad nacional ${ }^{3}$. EI SM es un conjunto de condiciones, interconectadas en sus aspectos bioquímicos, clínicos y metabólicos, que se ha relacionado con un incremento directo en el riesgo de enfermedades cardiovasculares y mortalidad ${ }^{4}$. Se ha definido como la combinación de obesidad central, alteración de la glucemia en ayunas, presión arterial elevada y dislipidemia con hipertrigliceridemia más valores bajos de colesterol unido a lipoproteínas de alta densidad (c-HDL, por las siglas en inglés de cholesterol-high density lipoprotein) ${ }^{5}$. Uno de los criterios más utilizados es el de la International Diabetes Federation, que toma en cuenta el perímetro abdominal para cada grupo étnico o raza, y para definir a un paciente con síndrome metabólico debe presentar obesidad central como principal requisito ${ }^{6,7}$. En la conjunción de la patogénesis de la osteoartritis y del SM se han implicado anormalidades que tienen en común alteraciones hormonales y en el metabolismo de la glucosa, resistencia a la insulina, factores de crecimiento, óxido nítrico y oxígeno ${ }^{8}$.

En la actualidad existe una creciente evidencia de que la obesidad es un síndrome complejo que conduce a una activación anormal del sistema neuroendocrino y de las vías proinflamatorias; de esta manera, la expansión del tejido adiposo aumenta la síntesis de citocinas proinflamatorias, como el factor de necrosis tumoral alfa y las interleucinas (IL) 1, 6, 8 y 18, disminuyendo además las citocinas reguladoras, como la IL-108-10. Un factor que también se ha implicado en la patogénesis del SM y de la gonartrosis es la proteína $C$ reactiva $(P C R)$, que es un marcador de inflamación inespecífico que se ha reportado incrementado en pacientes con obesidad; este incremento ha coincidido con una mayor prevalencia y progresión de la gonartrosis, así como de la coxartrosis. Se ha propuesto que estos sistemas de inflamación, en combinación con diabetes, resistencia a la insulina e hipertensión arterial, podrían aumentar el daño al cartílago y poner en riesgo los procesos de su reparación ${ }^{11,12}$. Por lo anterior, es necesario conocer si el SM y otros factores están relacionados con la gonartrosis, para posteriormente realizar estrategias preventivas y de control. Además, estos factores, solos o en su conjunto, podrían incrementar los índices de infección, trombosis arterial y muerte en los pacientes con gonartrosis que requieren cirugía de rodilla. El objetivo principal de este estudio fue estimar si existe asociación entre el SM y otros potenciales factores de riesgo con la presencia de gonartrosis.

\section{Método}

Se realizó un estudio transversal comparativo en el Servicio de Artroplastia de Rodilla de la Unidad Médica de Alta Especialidad Hospital de Traumatología y Ortopedia No. 21 del Instituto Mexicano del Seguro Social, en Monterrey, Nuevo León, México, durante el año 2016. Se calculó una muestra mediante la fórmula para comparar la proporción de dos poblaciones independientes $\left[n=\left(p_{1} q_{1}+p_{2} q_{2}\right)(k) /\left(p_{1}-p_{2}\right)^{2}\right]$ con una frecuencia esperada de SM en la población general del $35 \%$ y en pacientes con gonartrosis del $59 \%, 13,14$, resultando un mínimo de 49 pacientes por grupo. Se seleccionaron los pacientes del registro de consulta diaria en forma probabilística de acuerdo con el muestreo aleatorio del programa Open epi y que cumplieran los siguientes criterios: a) ser pacientes de primera vez, b) que acudieran a revisión por dolor de rodilla, y c) que contaran con radiografías anteroposteriores y laterales de rodilla. Se excluyeron pacientes con cirugías de reemplazo articular previas y pacientes con artropatías inflamatorias o traumáticas. Previo al inicio del estudio se realizaron reuniones de capacitación para los médicos tratantes participantes sobre el interrogatorio, la historia clínica y la medición antropométrica; se les dio a conocer el formato del instrumento de recolección de datos y una capacitación mensual a cada uno. Se realizó una prueba piloto antes del inicio de este estudio para evaluar la logística propuesta. Previo consentimiento informado se procedió al interrogatorio específico, el llenado de la hoja de registro de datos y la exploración física. El instrumento de captura de la información constó de dos secciones: la primera tuvo datos sociodemográficos, clínicos y de antecedentes patológicos; la segunda exploró los criterios para el diagnóstico de SM, mediciones de antropometría y resultados de laboratorio. Para la definición de SM se usó la escala de la International Diabetes Federation ${ }^{15}$, con obesidad 
central como principal requisito en relación con el grupo étnico al que se pertenece o un índice de masa corporal $\geq 30 \mathrm{~kg} / \mathrm{m}^{2}$, más dos de los siguientes cuatro factores: a) triglicéridos $\geq 150 \mathrm{mg} / \mathrm{dl}$ o tratamiento específico para esta anormalidad lipídica; b) c-HDL < $40 \mathrm{mg} / \mathrm{dl}$ en hombres y $<50 \mathrm{mg} / \mathrm{dl}$ en mujeres, o tratamiento específico para esta anormalidad lipídica; c) presión arterial sistólica $\geq 130 \mathrm{mmHg}$ o diastólica $\geq$ $85 \mathrm{mmHg}$, o tratamiento de hipertensión previamente diagnosticada; y d) glucosa plasmática en ayuno $\geq$ $100 \mathrm{mg} / \mathrm{dl}$ o diabetes tipo 2 previamente diagnosticada. La presencia de gonartrosis fue definida con la escala radiológica de Kellgren-Lawrence, con cambios radiográficos de grados 2,3 y $4^{16}$. Las radiografías se valoraron el día de la consulta, siendo examinadas por el médico tratante y un médico experto en forma ciega e independiente para corroborar el diagnóstico clínico y radiográfico de gonartrosis, obteniéndose una concordancia interobservador con un índice de Kappa ponderado de $0.64^{17}$. Se realizaron mediciones antropométricas de peso y talla, con ropa ligera y sin zapatos, en una báscula analógica calibrada con estadímetro. Se calculó el índice de masa corporal de acuerdo con la fórmula de Quetelet de la Organización Mundial de la Salud ${ }^{18}$. El perímetro abdominal se midió con cinta métrica flexible, con los brazos relajados a cada lado, en el punto que se encuentra entre la parte inferior de la última costilla y la parte más alta de la cadera en espiración. El perímetro de la cadera se midió con los brazos relajados a cada lado, en el lugar más ancho por encima de los glúteos sobre el pubis, y el índice cintura-cadera se obtuvo al dividir el perímetro de la cintura entre el perímetro de la cadera ${ }^{19}$. La presión arterial se midió con esfigmomanómetro calibrado estandarizado y estetoscopio de acuerdo con la auscultación de Korotkoff; se realizó en dos ocasiones con un reposo inicial estimado de 15 minutos y de 5 minutos entre cada medición. Se tomaron muestras de sangre en ayuno de las venas del pliegue del codo para determinaciones de glucosa, ácido úrico, PCR, triglicéridos, colesterol y perfil de lípidos, incluyendo lipoproteínas de alta, baja y muy baja densidad, que fueron procesadas mediante el equipo llab 650 IL (Instrumentation Laboratory SpA).

Para describir los datos se determinaron estadísticas como prevalencias, medias o medianas, y medidas de dispersión como desviación estándar o amplitud de intercuartil. Para el análisis comparativo se eligieron las pruebas $t$ de Student o $U$ de Mann-Whitney de acuerdo con los supuestos de normalidad y homocedasticidad; la prueba $\chi^{2}$ de Pearson se utilizó para evaluar frecuencias, y la odds ratio (OR) con su intervalo de confianza del 95\% (IC 95\%) para estimar asociación. Se realizó un análisis multivariado mediante el modelo de regresión logística para discriminar factores significativos. Se utilizó un valor de significación alfa $<0.05$.

\section{Resultados}

Se requirió evaluar a 350 candidatos, de los cuales $310(88.5 \%)$ cumplieron los criterios para ingresar. Se excluyeron 30 (8.5\%) pacientes porque no acudieron a completar sus exámenes de sangre y 10 (2.8\%) debido a que no se les tomó la totalidad de exámenes requeridos. La prevalencia de gonartrosis en la población total de este estudio fue del $80.3 \%$. Las características clínicas de los grupos de estudio se encuentran representadas en la tabla 1, en la que se aprecia que el síndrome metabólico en el grupo de pacientes con gonartrosis tuvo una prevalencia del $79.9 \%$ y en el grupo sin gonartrosis del $59 \%$, siendo una diferencia estadísticamente significativa $(p=0.001$; OR: 2.6; IC 95\%: 1.4-4.7). En el grupo de gonartrosis hubo un predominio de diabetes mellitus ( $p=0.02$; OR: 2.1; IC 95\%: 1.1-4) e hipertensión arterial sistémica ( $p=0.02$; OR: 1.9 ; IC 95\%: 1.1-3.3). No se encontraron diferencias estadísticamente significativas en nivel escolar, estado civil, ingesta de alcohol o tabaquismo. Al comparar las mediciones antropométricas en el grupo de pacientes con gonartrosis y sin gonartrosis, ninguna mostró tener diferencia estadística (tabla 2). De los resultados de laboratorio que se obtuvieron, la glucosa $(p=0.002)$ y el $c-H D L(p=0.01)$ tuvieron un valor estadísticamente más alto en el grupo de gonartrosis; el resto de los valores de laboratorio no tuvieron diferencias significativas entre los grupos estudiados (Tabla 3).

Se realizó un análisis multivariado mediante el modelo de regresión logística, en el cual se tomaron en cuenta las variables que fueron estadísticamente significativas para la existencia de gonartrosis en el análisis bivariado; en ese modelo, el SM fue el factor con mayor asociación para la presencia de gonartrosis $(p=0.003$; OR: 2.53, IC 95\%: 1.36-4.72), y el c-HDL mostró ser un factor relacionado con menor presencia de ella ( $p=0.022$; OR: 0.97, IC 95\%: 0.95-0.99) (Tabla 4).

\section{Discusión}

Una de las fortalezas que se encuentran en este estudio es que existen pocos reportes que hayan 
Tabla 1. Análisis comparativo de las características entre los grupos con y sin gonartrosis

\begin{tabular}{|c|c|c|c|c|c|}
\hline & $\begin{array}{c}\text { Con gonartrosis } n=249 \\
(80.3 \%)\end{array}$ & $\begin{array}{c}\text { Sin gonartrosis } \mathrm{n}=61 \\
(19.7 \%)\end{array}$ & OR & IC $95 \%$ & $p$ \\
\hline Edad & $66(60-72)^{*}$ & $68(61-75)^{*}$ & NA & NA & 0.23 \\
\hline Síndrome metabólico & $199(79.9 \%)$ & $36(59 \%)$ & 2.6 & $1.4-4.7$ & 0.001 \\
\hline Sexo femenino & $163(65.5 \%)$ & $39(63.9 \%)$ & 1.1 & $0.6-1.9$ & 0.82 \\
\hline Estudio $\leq 6$ años & $164(65.9 \%)$ & $36(59 \%)$ & 1.3 & $0.7-2.4$ & 0.31 \\
\hline No casado & $78(31.3 \%)$ & $20(32.8 \%)$ & 0.9 & $0.5-1.7$ & 0.82 \\
\hline Tabaquismo & $71(28.5 \%)$ & $18(29.5 \%)$ & 0.9 & $0.4-1$ & 0.87 \\
\hline Alcoholismo & $86(34.5 \%)$ & $26(42.6 \%)$ & 0.7 & $0.4-1.2$ & 0.23 \\
\hline Diabetes mellitus & $95(38.2 \%)$ & $14(23 \%)$ & 2.1 & $1.1-4.0$ & 0.02 \\
\hline HTA & $153(61.4 \%)$ & $28(45.9 \%)$ & 1.9 & $1.1-3.3$ & 0.02 \\
\hline Comorbilidad & $54(21.7 \%)$ & $14(23 \%)$ & 0.9 & $0.5-1.8$ & 0.83 \\
\hline \multicolumn{6}{|l|}{ IMC } \\
\hline$<25 \mathrm{~kg} / \mathrm{m}^{2}$ & $22(8.8 \%)$ & $7(11.5 \%)$ & - & - & \\
\hline $25-30 \mathrm{~kg} / \mathrm{m}^{2}$ & $68(27.3 \%)$ & $20(32.8 \%)$ & - & - & \\
\hline$>30 \mathrm{~kg} / \mathrm{m}^{2}$ & $159(63.9 \%)$ & $34(55.7 \%)$ & - & - & 0.19 \\
\hline
\end{tabular}

HTA: hipertensión arterial; IC 95\%: intervalo de confianza del 95\%; IMC: índice de masa corporal; NA: no aplica; OR: odds ratio.

Tabla 2. Comparación de las medidas antropométricas entre los grupos de estudio

\begin{tabular}{lccc}
\hline & $\begin{array}{c}\text { Con } \\
\text { gonartrosis } \\
\mathbf{n}=\mathbf{2 4 9}\end{array}$ & $\begin{array}{c}\text { Sin } \\
\text { gonartrosis } \\
\mathbf{n = 6 1}\end{array}$ & $\mathbf{p}$ \\
\hline Perímetro abdominal $(\mathrm{cm})$ & $105.5(96-113)$ & $103(93-110)$ & 0.16 \\
Índice cintura/cadera $(\mathrm{m})$ & $0.94 \pm 0.07$ & $0.93 \pm 0.08$ & 0.60 \\
Peso $(\mathrm{kg})$ & $77(71-89)$ & $77.5(70-88)$ & 0.56 \\
Talla $(\mathrm{m})$ & $1.56(1.51-1.62)$ & $1.59(1.52-1.66)$ & 0.87 \\
Perímetro de cadera $(\mathrm{cm})$ & $110(103-118)$ & $108.5(100-118)$ & 0.18 \\
PA sistólica $(\mathrm{mm} / \mathrm{Hg})$ & $140(120-150)$ & $130(120-140)$ & 0.29 \\
PA diastólica $(\mathrm{mm} / \mathrm{Hg})$ & $80(80-90)$ & $80(70-85)$ & 0.16 \\
\hline
\end{tabular}

PA: presión arterial.

Todos los valores, excepto el índice cintura/cadera, se describen con mediana y amplitud intercuartil.

estimado la magnitud del SM específicamente en pacientes con gonartrosis, y que se utilizó un muestreo probabilístico para disminuir el sesgo de selección. Por otra parte, una debilidad es que el lugar donde se llevó a cabo el estudio es un hospital de concentración donde predominan pacientes con edad avanzada y presencia o sospecha de patologías ortopédicas como gonartrosis, ocasionando que sea difícil hacer estudios analíticos que involucren una mayor proporción de participantes jóvenes y en
Tabla 3. Análisis de los resultados de laboratorio entre los pacientes con y sin gonartrosis

\begin{tabular}{lccc}
\hline & $\begin{array}{c}\text { Con } \\
\text { gonartrosis } \\
\mathbf{n}=\mathbf{2 4 9}\end{array}$ & $\begin{array}{c}\text { Sin } \\
\text { gonartrosis } \\
\mathbf{n = 6 1}\end{array}$ & $\mathbf{p}$ \\
\hline Ácido úrico (mg/dl) & $4.8(3.9-5.8)$ & $5.3(4.1-6.5)$ & 0.06 \\
Colesterol (mg/dl) & $190.5 \pm 40.9$ & $201.1 \pm 32.7$ & 0.06 \\
Glucosa (mg/dl) & $107(98-123)$ & $101(95-109)$ & 0.002 \\
Triglicéridos (mg/dl) & $131(104-181)$ & $119(97-157)$ & 0.13 \\
c-HDL (mg/dl) & $47(40-56)$ & $55(43-64)$ & 0.01 \\
C-LDL (mg/dl) & $110.7 \pm 29.7$ & $115.9 \pm 31.6$ & 0.22 \\
VLDL (mg/dl) & $26.6(21.2-37.2)$ & $26.3(19.8-38.6)$ & 0.40 \\
PCR (mg/l) & $0.0(0.0-1.6)$ & $0.1(0.0-2.5)$ & 0.44 \\
\hline
\end{tabular}

c-HDL: colesterol unido a lipoproteínas de alta densidad; c-LDL: colesterol unido a lipoproteínas de baja densidad; PCR: proteína C reactiva; VLDL: lipoproteínas de muy baja densidad.

Todos los valores, excepto el colesterol, se reportan con mediana y amplitud intercuartil.

estadios bajos del padecimiento, condicionando que la prevalencia de gonartrosis en este reporte fuera del $80.3 \%$, significativamente alta. Se han hecho diversas investigaciones en torno a la asociación que pudiera existir entre la gonartrosis y el SM, pero aún no hay evidencias sólidas de una asociación entre ambos padecimientos ${ }^{20}$. En este estudio, a pesar de que se utilizaron criterios estrictos para clasificar a los 
Tabla 4. Resultado del análisis multivariado mediante el modelo de regresión logística

\begin{tabular}{lccc}
\hline & $\mathbf{p}$ & OR & IC 95\% \\
\hline c-HDL & 0.022 & 0.97 & $0.95-0.99$ \\
Síndrome metabólico & 0.003 & 2.53 & $1.36-4.72$ \\
\hline
\end{tabular}

c-HDL: colesterol unido a lipoproteínas de alta densidad; IC 95\%: intervalo de confianza del 95\%; OR: odds ratio.

pacientes como portadores de $\mathrm{SM}^{21}$, su prevalencia en pacientes con y sin gonartrosis fue del 79.9 y el $59 \%$, respectivamente, siendo más altas que en otros reportes $^{3,22,23}$, posiblemente debido a que esos estudios fueron realizados con mayor población y en variados grupos de edad, y en cambio esta investigación tuvo una proporción alta de participantes de edad avanzada. Se han estudiado numerosos factores que pueden incidir en la aparición de gonartrosis $\mathrm{u}$ otros tipos de artrosis, como el sexo, la diabetes mellitus, la hipertensión arterial y el índice de masa corporal, pero hasta hace poco tiempo el SM en su conjunto no había sido investigado exhaustivamente como un posible factor asociado $0^{5,12,24,25}$. En este estudio, el SM y algunos de sus componentes mostraron ser más prevalentes en estadios de degeneración articular, similar a lo observado por Yoshimura, et al. ${ }^{24}$, aunque ellos encontraron otros factores de progresión a gonartrosis más grave, como el sexo femenino y la edad ${ }^{24}$, distinto a lo encontrado en este trabajo, en el cual el sexo femenino y la edad no mostraron ser factores asociados a gonartrosis, esto último posiblemente por el predominio ya referido de pacientes con edad avanzada de la muestra. En esta investigación se tuvo una prevalencia alta de pacientes con diabetes mellitus, hipertensión arterial y obesidad, condiciones todas ellas también relacionadas con poblaciones de mayor edad. En los valores de laboratorio que se tomaron en consideración para comparar los grupos de pacientes con y sin gonartrosis se encontraron diferencias significativas a favor de un mayor valor de glucosa en el grupo de gonartrosis, en concordancia con una mayor prevalencia de diabetes en ese mismo grupo. Mayores valores de c-HDL mostraron estar relacionados con una menor presencia de gonartrosis, hallazgo que está en concordancia con lo esperado, ya que el SM, el cual se encontró asociado a mayor gonartrosis en el mismo análisis, se caracteriza por valores bajos de c-HDL. Estudios bioquímicos y celulares sugieren que todos estos factores pudieran estar implicados en la patogénesis de la degeneración del cartílago articular en conjunto con la obesidad, y que se relacionan con la secreción de citocinas proinflamatorias, las cuales pudieran ser marcadores útiles de progresión a degeneración articular ${ }^{10,26-28}$. Debido a esto se analizaron los valores de PCR, que es un marcador de inflamación inespecífico y que se ha reportado aumentado en los pacientes con obesidad y SM. En este reporte no se detectaron diferencias significativas en cuanto a la PCR entre los pacientes con y sin gonartrosis, en concordancia con el estudio de Engström, et al. ${ }^{11}$, en el que se muestra que, después de ajustar los factores confusores, la PCR no se relacionó con un aumento en la incidencia de gonartrosis. Dentro de los resultados encontrados en esta investigación se observan algunas consistencias respecto a lo referido por $\mathrm{Shin}^{29}$, mostrando una asociación del SM con cambios radiográficos de degeneración articular más grave; sin embargo, él también aprecia una correlación con la obesidad, contrario a este estudio, en el cual las mediciones antropométricas, especialmente el índice cintura-cadera, no tuvieron diferencias significativas entre los grupos con y sin gonartrosis $(p=$ 0.6). Lo anterior también difiere del trabajo realizado por Gandhi et al. ${ }^{30}$, el cual muestra que en las mujeres con estadios más avanzados de gonartrosis predomina un mayor índice cintura-cadera ${ }^{30}$.

En conclusión, el SM y las alteraciones que lo componen pueden ser factores implicados en la aparición y la progresión de gonartrosis, y su control pudiera prevenir su aparición. El SM puede aumentar hasta más de dos veces el riesgo de presentar gonartrosis.

\section{Bibliografía}

1. Inoue R, Ishibashi $Y$, Tsuda E, et al. Medical problems and risk factors of metabolic syndrome among radiographic knee osteoarthritis patients in the Japanese general population. J Orthop Sci Off J Jpn Orthop Assoc. 2011;16:704-9.

2. Zhang $Y$, Jordan JM. Epidemiology of osteoarthritis. Clin Geriatr Med. 2010;26:355-69.

3. Puenpatom RA, Victor TW. Increased prevalence of metabolic syndrome in individuals with osteoarthritis: an analysis of NHANES III data. Postgrad Med. 2009;121:9-20.

4. Kaur J, Kaur J. A comprehensive review on metabolic syndrome. Cardiol Res Pract Cardiol Res Pract. 2014;2014:943162.

5. Yoshimura N, Muraki S, Oka H, et al. Association of knee osteoarthritis with the accumulation of metabolic risk factors such as overweight, hypertension, dyslipidemia, and impaired glucose tolerance in Japanese men and women: the ROAD study. J Rheumatol. 2011;38:921-30.

6. Khosravi-Boroujeni $H$, Ahmed F, Sadeghi M, et al. Does the impact of metabolic syndrome on cardiovascular events vary by using different definitions? BMC Public Health. 2015;15:1313.

7. Gandhi R, Razak F, Tso P, et al. Asian ethnicity and the prevalence of metabolic syndrome in the osteoarthritic total knee arthroplasty population. J Arthroplasty. 2010;25:416-9.

8. Heidari B. Knee osteoarthritis prevalence, risk factors, pathogenesis and features: part I. Casp J Intern Med. 2011;2:205-12.

9. Han CD, Yang IH, Lee WS, et al. Correlation between metabolic syndrome and knee osteoarthritis: data from the Korean National Health and Nutrition Examination Survey (KNHANES). BMC Public Health. 2013;13:603. 
10. Iannone F, Lapadula G. Obesity and inflammation - targets for OA therapy. Curr Drug Targets. 2010;11:586-98.

11. Engström G, Gerhardsson de Verdier M, Rollof J, et al. C-reactive protein, metabolic syndrome and incidence of severe hip and knee osteoarthritis. A population-based cohort study. Osteoarthr Cartil OARS Osteoarthr Res Soc. 2009;17:168-73.

12. Maddah S, Mahdizadeh J. Association of metabolic syndrome and its components with knee osteoarthritis. Acta Med Iran. 2015;53:743-8.

13. Aguilar M, Bhuket $\mathrm{T}$, Torres $\mathrm{S}$, et al. Prevalence of the metabolic syndrome in the united states, 2003-2012. JAMA. 2015;313:1973-4.

14. Rojas-Martínez R, Aguilar-Salinas CA, Jiménez-Corona A, et al. Prevalence of obesity and metabolic syndrome components in Mexican adults without type 2 diabetes or hypertension. Salud Pública México. 2012;54:7-12.

15. International Diabetes Federation. IDF Worldwide Definition of the Metabolic Syndrome. Disponible en: http://www.idf.org/metabolic-syndrome

16. Kellgren JH, Lawrence JS. Radiological assessment of osteo-arthrosis. Ann Rheum Dis. 1957:16:494-502.

17. Riddle DL, Jiranek WA, Hull JR. Validity and reliability of radiographic knee osteoarthritis measures by arthroplasty surgeons. Orthopedics. 2013;36:e25-32.

18. World Health Organization. Obesity: preventing and managing the global epidemic. Report of a WHO consultation. World Health Organization technical report series. 2000;894:i-xii, 1-253.

19. World Health Organization. WHO STEPwise approach to surveillance (STEPS). Disponible en: http://www.who.int/chp/steps/en

20. Le Clanche S, Bonnefont-Rousselot D, Sari-Ali E, et al. Inter-relations between osteoarthritis and metabolic syndrome: a common link? Biochimie. 2016;121:238-52.
21. Khosravi-Boroujeni $\mathrm{H}$, Ahmed $\mathrm{F}$, Sadeghi $\mathrm{M}$, et al. Does the impact of metabolic syndrome on cardiovascular events vary by using different definitions? BMC Public Health. 2015;15:1313.

22. Lee G-C. What's new in adult reconstructive knee surgery. J Bone Joint Surg Am. 2016:98:156-65.

23. Šalamon L, Morović-Vergles J, Marasović-Krstulović D, et al. Differences in the prevalence and characteristics of metabolic syndrome in rheumatoid arthritis and osteoarthritis: a multicentric study. Rheumatol Int. 2015;35:2047-57.

24. Yoshimura N, Muraki S, Oka H, et al. Accumulation of metabolic risk factors such as overweight, hypertension, dyslipidaemia, and impaired glucose tolerance raises the risk of occurrence and progression of knee osteoarthritis: a 3-year follow-up of the ROAD study. Osteoarthritis and Cartilage. 2012;20:1217-26.

25. Bruyère $\mathrm{O}$ Cooper $\mathrm{C}$, Arden $\mathrm{N}$, et al. Can we identify patients with high risk of osteoarthritis progression who will respond to treatment? A focus on epidemiology and phenotype of osteoarthritis. Drugs Aging. 2015;32:179-87.

26. Kluzek S, Newton JL, Arden NK. Is osteoarthritis a metabolic disorder? Br Med Bull. 2015;115:111-21.

27. Berenbaum F, Eymard F, Houard X. Osteoarthritis, inflammation and obesity. Curr Opin Rheumatol. 2013;25:114-8.

28. Zhuo Q, Yang W, Chen J, et al. Metabolic syndrome meets osteoarthritis. Nat Rev Rheumatol. 2012;8:729-37.

29. Shin D. Association between metabolic syndrome, radiographic knee osteoarthritis, and intensity of knee pain: results of a national survey. J Clin Endocrinol Metab. 2014:99:3177-83.

30. Gandhi R, Dhotar H, Tsvetkov D, et al. The relation between body mass index and waist-hip ratio in knee osteoarthritis. Can J Surg J Can Chir. 2010;53:151-4. 